\title{
Cisplatin resistant ovarian cancer cells reveal a polyploid phenotype with remarkable activation of nuclear processes
}

\section{Rezvan Adibi}

Isfahan University of Medical Sciences

Shiva Moein

Regenerative Medicine Research Center, Isfahan University of Medical Sciences

Yousof Gheisari ( $\nabla$ ygheisari@med.mui.ac.ir)

Isfahan University of Medical Sciences

\section{Research Article}

Keywords: Ovary cancer, Polyploid Cells, Cancer resistance, Gene expression profiling, Systems biology

Posted Date: May 3rd, 2021

DOl: https://doi.org/10.21203/rs.3.rs-440506/v1

License: (1) This work is licensed under a Creative Commons Attribution 4.0 International License.

Read Full License 


\section{Abstract}

Background Tumor recurrence as a main cause of cancer death is a big barrier in cancer complete treatment. Various studies denote for the possible role of therapeutics in tumor relapse. Cisplatin as one of the generally-used chemotherapy agents is supposed to be the source of therapy-resistance through formation of polyploid giant cancer cells (PGCCs). Nevertheless, the mechanisms by which PGCCs promote tumor relapse are not fully understood.

Methods In this study we performed experimental and bioinformatic investigations to recognize the mechanisms related to cisplatin resistance. A2780 and SCOV-3 cell lines were treated with cisplatin for 72 hours and were evaluated for their morphology by fluorescent microscopy and DNA content analysis. Furthermore, a microarray dataset of cisplatin-resistant ovarian cancer cells was reanalyzed to determine the significantly altered genes and signaling pathways.

Result Although cisplatin led to death of considerable fraction of cells in both cell lines, a significant number of survived cells became polyploid. On the other hand, our high throughput analysis determined significant change in expression of 1930 genes which mainly related to gene regulatory mechanisms and nuclear processes. Besides, mTOR, hypoxia, Hippo and 14-3-3 signaling pathways previously shown to have role in PGCCs were determined.

Conclusion Taken together, results of this study demonstrated some key biological mechanisms related to cisplatin-resistant polyploid cancer cells.

\section{Background}

As the second leading cause of death, cancer is one of the most problematic health issues all over the world. Despite huge investigations, drug resistance and tumor relapse after chemo/radiotherapy have remained prominent barriers in cancer treatment [1]. This indicates that the main elements contributing to these processes are not yet completely understood. For two centuries, numerous studies have shown the contribution of polyploid giant cancer cells (PGCCs) in cancer progression [2]. In this paradigm, the polyploid cancer cells with the capability of breeding cancer stem cells are tumor generators posttreatment and are the main cause of therapy resistance and tumor recurrence $[3,4]$.

Cell polyploidy is not restricted to cancers, but an evolutionarily conserved mechanism to support tissue regeneration and combat against stressors [5]. Formation of PGCCs can be induced by either natural tensions such as hypoxia, acidic condition, and mechanical pressures or by chemotherapy and radiotherapy [6-9]. The survived polyploid cells then serve as a source for the generation of new tumor cells and hence "tumor regeneration" [10]. Despite various reports on the association of these cells with tumor resistance and relapse, little is known about the molecular pathways related to cell polyploidy. A study by Sharma et al. determined that the MTOR signaling pathway, a known regulator of cell metabolism, plays an important role in the polyploidy state in cancer cells. Moreover, Other studies have 
also shown the importance of HIPPO, 14-3-3 and Hypoxia signaling pathways in the induction of polyploidy in tumor cells [11-15].

The recent emergence of the systems biology approach has promoted the investigation of cancer polyploidy. Rantala et al. demonstrated the role of GINS2 as a regulator of the DNA damage repair system by analysis of the transcriptome profile of breast cancer samples after targeting genes by a siRNA library [16]. In another study, Jung et al. explored changes in proteome and phospho-proteome of Cisplatinresistant bladder cancer cells which are related to activation of polyploidy in cancer cells [17]. Despite these findings, our knowledge of the underlying molecular mechanisms regulating the emergence and function of PGCCs is in its infancy.

Cisplatin is a common chemotherapy reagent that has been used for a wide variety of malignancies and is associated with a high rate of relapse [18]. To investigate the role of polyploidy in cisplatin resistance, two ovarian cancer cell lines were followed upon drug administration. In parallel, using high throughput transcriptomic data, the molecular machinery activated in cisplatin-resistance polyploid cells was investigated. Results of this study unraveled new signaling pathways related to polyploidization of cancer cells.

\section{Methods}

\section{Cell culture}

Ovarian cancer cell lines, A2780 and SKOV3 were purchased from Pasteur Institute of Iran. The cells were cultured in Dulbecco's Eagle's minimal essential medium (DMEM) enriched with 10\% FBS and 1\% Penicillin-Streptomycin (Bioidea, Iran) and incubated in $37^{\circ} \mathrm{C}$ and $5 \% \mathrm{CO} 2$.

\section{Induction of polyploid giant cancer cells}

In order to determine the optimum dose of cisplatin (Mylan, France) which induces the highest percentage of polyploidy, the cells were treated with $3,6,13,18,50,70$ and $100 \mu \mathrm{M}$ of the drug. DNA content analysis determined that $6 \mu \mathrm{M}$ concentration of the drug, has the most ability in induction of polyploidy in both cell lines (data not shown). Cisplatin was added to the culture medium for 72 hours and then thoroughly washed by PBS (Gibco, USA). 7 days post drug administration, the cells were evaluated for their morphologic features by light and fluorescent microscopy as well as DNA content measurement.

\section{DNA content analysis}

The cells were fixed with $70 \%$ ethanol and kept in $-20^{\circ} \mathrm{C}$ overnight. Subsequently, the fixed cells were treated with PI (Sigma, Germany)/Triton X-100 (Cytomatin gene, Iran)/ RNase A (Sigma, Germany) solution for 30 min and were read by FACS Calibur instrument (BD Biosciences, USA). Flow cytometry results were analyzed using flow jo software version 10.6.2.

\section{DAPI staining}


For fluorescent staining of the genome content, the cells were fixed with paraformaldehyde $4 \%$ for 20 minutes and washed twice with PBS. Subsequently the cells were permeabilized by $0.04 \%$ Triton X-100 and stained with 40,6-diamidino-2-phenylindole dihydrochloride (DAPI) (Sigma, Germany) at the concentration of $1 \mu \mathrm{g} / \mathrm{ml}$. Fluorescent microscopy and imaging were performed using Nikon Eclipse Ti (Nikon, Japan).

\section{Microarray data analysis}

The dataset GSE58470 which contains the gene expression profile of IGROV-1 cisplatin-resistant ovarian cancer cell line and control group was obtained from the Gene Expression Omnibus (GEO) database. The Quality of the data was assessed by principal component analysis (PCA), hierarchical clustering and volcano plots using ggplot2 package of the $\mathrm{R}$ software (Version 3.5.1). Differentially expressed genes were determined by GEO2R tool of the GEO database. Benjamini-Hochberg false discovery rate (FDR) was used as the $p$-value correction method and all the genes with adjusted $p$-value $<0.05$ were assumed as DEGs.

\section{Pathway enrichment analysis}

Pathway enrichment analysis was performed by Ingenuity pathway Analysis (IPA) (Qiagen Inc.) [19]. Directional changes with Z-score $>2$ were considered as significant alteration activities. MetaboAnalyst software (version 3.0) was also applied for pathway enrichment analysis and significant pathways (adjusted $p$-value $<0.05$ ) were determined by fisher exact test.

\section{PPI (Protein-Protein Interaction) network construction and module identification}

PPI Network was constructed by CluePedia plugin version 1.5.7 of Cytoscape version 3.8.0. The topology of the network was analyzed by Cytoscape Network Analyzer tool and nodes with the highest degree and betweenness centrality parameters were identified. Structural modules of the PPI network based on clustering coefficient were determined by MCODE plugin version 1.32 of the Cytoscape software [20].

\section{Results}

\section{Cisplatin enriches polyploidy in ovarian cancer cell lines}

Considering that polyploidy is proposed as a leading player in chemo-drug resistance [10], the effect of cisplatin was examined on two ovarian cancer cell lines. A2789 and SCOV-3 cells were treated with cisplatin for 72 hours and then recovered for 7 days. As expected, cisplatin resulted in massive cell death. Notably, the surviving sub-population was enriched for polyploid cells as revealed by fluorescent microscopy following DNA-staining. Indeed, PGCCs as large as $100 \mu \mathrm{m}$ were evident in the culture of cisplatin-treated A2789 and SCOV-3 lines. For further validation and quantification, DNA content analysis was performed which demonstrated 2.0- and 4.7-fold enhancement in the ratio of PGCCs in A2789 and SCOV-3 cells, respectively (Fig. 1). This finding suggests that polyploid cells can be a source of cisplatinresistance in ovarian cancer. 


\section{Transcriptomics profile of cisplatin-resistant ovarian cancer cells highlights the role of nuclear processes}

To investigate molecular mechanisms involved in polyploid-resistant cells, the GSE58470 microarray dataset originally generated by Cossa et al. [21, 22] was reanalyzed. They established a cisplatin-resistant ovarian cancer line and performed mRNA microarray profiling concluding that the ERK $1 / 2$ pathway has an important role in cisplatin resistance.

Re-analysis of this dataset was started with a quality control assessment. As previously shown, about half of the publicly available transcriptomics profiles suffer from the lack of sufficient quality, underscoring the importance of pre-assessments of such data [23]. PCA and hierarchical clustering denoted the acceptable quality of the selected dataset as the samples were separated according to experimental groups in an unsupervised manner (Fig. 2a, b). A comparison of cisplatin-resistant and control group determined 1930 DEGs with at least two-fold overexpression or down-regulation (|log2FC| $\geq 1$ ) and adjusted $p$-value $<0.05$ (Fig. 2c).

To assess the functional role of the identified DEGs, pathway enrichment analysis was performed determining the 21 signaling pathways with $p$-value $<0.05$ (Fig. 3a). Among them, the protein ubiquitination pathway, FAT10 signaling pathway, and phagosome maturation have been previously reported to be involved in chemotherapy resistance [24-26]. Moreover, mTOR, 14-3-3, HIPPO and Hypoxia are known as polyploidy-related pathways $[12,13,15,27]$. Additionally, enrichment for biological process and molecular function $\mathrm{GO}$ terms highlighted RNA polymerase regulation and protein metabolism. Noteworthy, nucleoplasm was an enriched cellular component term with the maximum number of child terms (Fig. 3b). Overall, these findings indicate that gene regulation mechanisms and nucleus processes are critical for drug resistance and potentially cancer polyploidy.

The interaction network was constructed to identify the map of communications between the identified DEGs. To identify the most influential genes in the network, centrality analysis was performed and top 10 genes with the highest degree and betweenness centrality measures were detected (Fig. 4a). A considerable fraction of these central genes is involved in nuclear processes; POLR2C, RANBP2, UPF3B, $S P 1$ and $E L O B$ contributed to RNA metabolism and gene expression mechanisms. Also, a group of central genes including $U B C, U B E 2 D 2$ and $S U M O 1$ are involved in protein ubiquitination. Previous studies have also suggested the role of PLK1 in the cell cycle regulation of PGCCs [28].

Densely connected regions of the constructed network, known as structural modules were determined based on clustering coefficient centrality feature. These modules were mainly related to RNA metabolism, protein localization, chromatid segregation, microtubule polymerization and membrane budding (Fig. 4b).

\section{Discussion}

Cancer resistance to therapy was the cause of 10 million deaths in 2020 [29]. For decades, cancer researchers have tried to recognize the source of this resistance and find effective ways to combat 
cancer. In 1858, Virchow reported large nuclei and nucleoli in cancer cells [30] and in 1968, Bauke \& Schöffling provided the first clinical evidence on the correlation between cancer growth and polyploid giant cancer cells [31]. Since then, despite opposite opinions [32], PGCCs have been considered a source of chemo/radio therapy resistance $[6,8,33]$. PGCCs and their offspring cells have been also shown to possess stem cell characteristics. For instance, Zhang et al. and Niu et al. revealed PGCCs and their offspring could express stem cell markers such as OCT4, NANOG, and SOX2 [6, 7]. Despite these investigations, PGCCs have rarely been approached as a therapeutic target, potentially due to the lack of sufficient evidence on their molecular biology. Here, an in vitro model of PGCCs was established and gene expression patterns associated with chemotherapy resistance were explored.

Cisplatin is one the most broad-spectrum chemotherapy agents and the first-line therapy in ovarian cancer [34]. This study has provided evidence showing the effect of this drug on two ovarian cancer cell lines, A2780 and SCOV-3. Seven days post cisplatin administration, a subpopulation of large cells with several nuclei was remarkably observed in fluorescent microscopy inspections. In agreement, DNA content analysis demonstrated a significant increment in the percentage of polyploid cells in both cell lines after exposure to cisplatin.

The comprehensive viewpoint of systems biology approaches would be useful in deciphering the features of these cells and providing a holistic map of their functions. In this study, reanalysis of a transcriptome microarray dataset belonging to cisplatin-resistant ovarian cells determined critical role of various signaling pathways in promotion of cancer resistance. Among the pathways, the role of mTOR, 14-3-3, HIPPO, and Hypoxia signaling pathways are previously identified in PGCCs. Similarly, Liu et al. have shown that inhibition of the mTOR pathway with rapamycin reduced polyploidy in leukemic cells. Additionally, Sharma et al. demonstrated that mTOR inhibitor AZD8055 could decrease the formation of cancer polyploid cells and increase chemosensitivity in breast cancer $[15,35]$. It is also shown that overexpression of 14-3-3, known as a regulator of the cell cycle, caused the emergence of polyploidy in non-small cell lung cancer [12]. Moreover, Zhang et al. demonstrated that activation of the Hippo signaling pathway via the Akt-skp2 axis promotes cell cycle arrest and polyploidy in hepatocytes and hepatocellular carcinoma $[11,27]$. Furthermore, a bundle of evidence highlights the role of natural or simulated hypoxic stresses in the induction of polyploidy in cancer cells $[6,13,36,37]$. Other signaling pathways enriched in our analysis may also play role in cancer polyploidy. Accordingly, the protein ubiquitination pathway which is highlighted in our study has previously shown to be involved in DNA damage repair, tumor proliferation, hypoxia adaptation, and cancer stem cell modulation to chemotherapy resistance [26, 38-43]. Also, several recent studies demonstrated the role of FAT10 in chemoresistance [24] and epithelial to mesenchymal transition [25], both of which are associated with cancer polyploidy [44].

Among 1930 DEGs that were identified, most of them belonged to gene regulation and nuclear processes according to $\mathrm{GO}$ enrichment analysis. As well, the top central genes of the constructed network typically attributed to nuclear process. According to our previous studies indicating the importance of central genes in promotion of cellular phenotype $[45,46]$, we think that the central nodes as well as genes in the 
densely-connected modules which belonged to chromatid segregation, microtubule polymerization and membrane budding, indicate the key role of nuclear processes and polyploidy in cancer resistance [4754].

\section{Conclusions}

Taken together, this study provides insights on key molecular processes involved in chemotherapy resistance and highlights the role of PGCCs. Further studies are required to elucidate the underlying mechanisms and pave the way for future therapeutic strategies.

\section{Abbreviations}

PGCC: Polyploid Giant Cancer Cell; IPA: Ingenuity Pathway Analysis; PCA: Principal Component Analysis; GEO: Gene Expression Omnibus; DEGs: Differentially Expressed Genes; PPI: Protein-Protein Interaction

\section{Declarations}

\section{Acknowledgements}

We thank Amir Bahreini and Yasin Eshraghi for their assistance in interpretation of the microarray data using IPA software.

Some of the results of this study were presented in the ROYAN international congress 2019 as poster.

\section{Authors' contributions}

R.A., S.M., and Y.G. conceived the study protocol. R.A. performed the experiments, processed the data and wrote the manuscript. S.M. and Y.G. revised the manuscript. All authors made a substantial intellectual contribution to the work, and approved the submitted version for publication.

\section{Funding}

This study was supported by Isfahan University of Medical Sciences (grant number: 51036) and Student Research Committee of Isfahan University of Medical Sciences (grant number: 199485)

\section{Ethics approval and consent to participate}

No ethics approval was required for this work. All data in this study are publicly available.

\section{Consent for publication}

Not applicable.

\section{Competing interests}


The authors declare that they have no competing interests.

\section{Availability of data and materials}

The microarray dataset reanalyzed in this study can be downloaded from GEO data repository with accession number GSE58470.

\section{References}

1. Flemming A. Cancer stem cells: Targeting the root of cancer relapse. Nature reviews Drug discovery. 2015;14(3):165.

2. Amend SR, Torga G, Lin KC, Kostecka LG, de Marzo A, Austin RH, et al. Polyploid giant cancer cells: Unrecognized actuators of tumorigenesis, metastasis, and resistance. Prostate. 2019;79(13):148997.

3. Ayob AZ, Ramasamy TS. Cancer stem cells as key drivers of tumour progression. J Biomed Sci. 2018;25(1):20.

4. Batlle E, Clevers H. Cancer stem cells revisited. Nat Med. 2017;23(10):1124-34.

5. Øvrebø $\mathrm{Jl}$, Edgar BA. Polyploidy in tissue homeostasis and regeneration. Development (Cambridge, England). 2018;145(14).

6. Zhang S, Mercado-Uribe I, Xing Z, Sun B, Kuang J, Liu J. Generation of cancer stem-like cells through the formation of polyploid giant cancer cells. Oncogene. 2014;33(1):116-28.

7. Niu N, Mercado-Uribe I, Liu J. Dedifferentiation into blastomere-like cancer stem cells via formation of polyploid giant cancer cells. Oncogene. 2017;36(34):4887-900.

8. Puig L, Fernandez-Figueras MT, Bielsa I, Lloveras B, Alomar A. Multinucleate cell angiohistiocytoma: a fibrohistiocytic proliferation with increased mast cell numbers and vascular hyperplasia. J Cutan Pathol. 2002;29(4):232-7.

9. Diaz-Carballo D, Saka S, Klein J, Rennkamp T, Acikelli AH, Malak S, et al. A Distinct Oncogenerative Multinucleated Cancer Cell Serves as a Source of Stemness and Tumor Heterogeneity. Cancer research. 2018;78(9):2318-31.

10. Moein S, Adibi R, da Silva Meirelles L, Nardi NB, Gheisari Y. Cancer regeneration: Polyploid cells are the key drivers of tumor progression. Biochim Biophys Acta Rev Cancer. 2020;1874(2):188408.

11. Zhang S, Chen Q, Liu Q, Li Y, Sun X, Hong L, et al. Hippo Signaling Suppresses Cell Ploidy and Tumorigenesis through Skp2. Cancer Cell. 2017;31(5):669-84.e7.

12. Gomes CJ, Centuori SM, Harman MW, Putnam CW, Wolgemuth CW, Martinez JD. The induction of endoreduplication and polyploidy by elevated expression of 14-3-3gamma. Genes \& cancer. 2017;8(11-12):771-83.

13. Lopez-Sanchez LM, Jimenez C, Valverde A, Hernandez V, Penarando J, Martinez A, et al. CoCl2, a mimic of hypoxia, induces formation of polyploid giant cells with stem characteristics in colon cancer. PloS one. 2014;9(6):e99143. 
14. Spankuch-Schmitt B, Wolf G, Solbach C, Loibl S, Knecht R, Stegmuller M, et al. Downregulation of human polo-like kinase activity by antisense oligonucleotides induces growth inhibition in cancer cells. Oncogene. 2002;21(20):3162-71.

15. Sharma S, Yao HP, Zhou YQ, Zhou J, Zhang R, Wang MH. Prevention of BMS-777607-induced polyploidy/senescence by mTOR inhibitor AZD8055 sensitizes breast cancer cells to cytotoxic chemotherapeutics. Molecular oncology. 2014;8(3):469-82.

16. Rantala JK, Edgren H, Lehtinen L, Wolf M, Kleivi K, Vollan HK, et al. Integrative functional genomics analysis of sustained polyploidy phenotypes in breast cancer cells identifies an oncogenic profile for GINS2. Neoplasia (New York, NY). 2010;12(11):877-88.

17. Jung JH, You S, Oh JW, Yoon J, Yeon A, Shahid M, et al. Integrated proteomic and phosphoproteomic analyses of cisplatin-sensitive and resistant bladder cancer cells reveal CDK2 network as a key therapeutic target. Cancer letters. 2018;437:1-12.

18. Dasari S, Tchounwou PB. Cisplatin in cancer therapy: molecular mechanisms of action. Eur $\mathrm{J}$ Pharmacol. 2014;740:364-78.

19. Krämer A, Green J, Pollard J, Jr., Tugendreich S. Causal analysis approaches in Ingenuity Pathway Analysis. Bioinformatics (Oxford, England). 2014;30(4):523-30.

20. Bader GD, Hogue CW. An automated method for finding molecular complexes in large protein interaction networks. BMC bioinformatics. 2003;4:2.

21. Cossa G, Lanzi C, Cassinelli G, Carenini N, Arrighetti N, Gatti L, et al. Differential outcome of MEK1/2 inhibitor-platinum combinations in platinum-sensitive and -resistant ovarian carcinoma cells. Cancer letters. 2014;347(2):212-24.

22. Arrighetti N, Cossa G, De Cecco L, Stucchi S, Carenini N, Corna E, et al. PKC-alpha modulation by miR483-3p in platinum-resistant ovarian carcinoma cells. Toxicology and applied pharmacology. 2016;310:9-19.

23. Abedi M, Fatehi R, Moradzadeh $\mathrm{K}$, Gheisari Y. Big data to knowledge: common pitfalls in transcriptomics data analysis and representation. RNA biology. 2019;16(11):1531-3.

24. Xue F, Zhu L, Meng QW, Wang L, Chen XS, Zhao YB, et al. FAT10 is associated with the malignancy and drug resistance of non-small-cell lung cancer. OncoTargets and therapy. 2016;9:4397-409.

25. Li C, Wang Z, Feng N, Dong J, Deng X, Yue Y, et al. Human HLA-F adjacent transcript 10 promotes the formation of cancer initiating cells and cisplatin resistance in bladder cancer. Molecular medicine reports. 2018;18(1):308-14.

26. Gallo LH, Ko J, Donoghue DJ. The importance of regulatory ubiquitination in cancer and metastasis. Cell cycle (Georgetown, Tex). 2017;16(7):634-48.

27. Loss of Hippo Signaling Promotes Polyploidy and Tumorigenesis. 2017;7(7):661-.

28. Jemaà M, Kifagi C, Serrano SS, Massoumi R. Preferential Killing of Tetraploid Colon Cancer Cells by Targeting the Mitotic Kinase PLK1. Cellular physiology and biochemistry : international journal of experimental cellular physiology, biochemistry, and pharmacology. 2020;54(2):303-20. 
29. Sung H, Ferlay J, Siegel RL, Laversanne M, Soerjomataram I, Jemal A, et al. Global cancer statistics 2020: GLOBOCAN estimates of incidence and mortality worldwide for 36 cancers in 185 countries. CA: a cancer journal for clinicians. 2021.

30. Virchow R. Cellular pathology. As based upon physiological and pathological histology. Lecture XVI-Atheromatous affection of arteries. 1858. Nutrition reviews. 1989;47(1):23-5.

31. Bauke J, Schöffling K. Polyploidy in human malignancy. Hypopentaploid chromosome pattern in malignant reticulosis with secondary sideroachrestic anemia. Cancer. 1968;22(3):686-94.

32. Nair JS, Ho AL, Schwartz GK. The induction of polyploidy or apoptosis by the Aurora A kinase inhibitor MK8745 is p53-dependent. Cell cycle (Georgetown, Tex). 2012;11(4):807-17.

33. Bell AG, Baker DG. X-IRRADIATION-INDUCED POLYPLOIDY IN HUMAN LEUCOCYTE CULTURES. Experimental cell research. 1965;38:144-52.

34. Bergamini A, Pisano C, Di Napoli M, Arenare L, Della Pepa C, Tambaro R, et al. Cisplatin can be safely administered to ovarian cancer patients with hypersensitivity to carboplatin. Gynecologic oncology. 2017; 144(1):72-6.

35. Sharma S, Zeng JY, Zhuang CM, Zhou YQ, Yao HP, Hu X, et al. Small-molecule inhibitor BMS-777607 induces breast cancer cell polyploidy with increased resistance to cytotoxic chemotherapy agents. Mol Cancer Ther. 2013;12(5):725-36.

36. Rofstad EK, Johnsen NM, Lyng H. Hypoxia-induced tetraploidisation of a diploid human melanoma cell line in vitro. The British journal of cancer Supplement. 1996;27:S136-9.

37. Braune EB, Tsoi YL, Phoon YP, Landor S, Silva Cascales H, Ramsköld D, et al. Loss of CSL Unlocks a Hypoxic Response and Enhanced Tumor Growth Potential in Breast Cancer Cells. Stem cell reports. 2016;6(5):643-51.

38. Wu X, Zhang W, Font-Burgada J, Palmer T, Hamil AS, Biswas SK, et al. Ubiquitin-conjugating enzyme Ubc13 controls breast cancer metastasis through a TAK1-p38 MAP kinase cascade. Proceedings of the National Academy of Sciences of the United States of America. 2014;111(38):13870-5.

39. Sun T, Liu Z, Yang Q. The role of ubiquitination and deubiquitination in cancer metabolism. Molecular cancer. 2020;19(1):146.

40. Messick TE, Greenberg RA. The ubiquitin landscape at DNA double-strand breaks. The Journal of cell biology. 2009;187(3):319-26.

41. Altun M, Zhao B, Velasco K, Liu H, Hassink G, Paschke J, et al. Ubiquitin-specific protease 19 (USP19) regulates hypoxia-inducible factor $1 \mathrm{a}(\mathrm{HIF-1a})$ during hypoxia. The Journal of biological chemistry. 2012;287(3):1962-9.

42. Brahimi-Horn $\mathrm{C}$, Pouysségur $\mathrm{J}$. When hypoxia signalling meets the ubiquitin-proteasomal pathway, new targets for cancer therapy. Critical reviews in oncology/hematology. 2005;53(2):115-23.

43. Yang L, Shi P, Zhao G, Xu J, Peng W, Zhang J, et al. Targeting cancer stem cell pathways for cancer therapy. Signal transduction and targeted therapy. 2020;5(1):8. 
44. Wang X, Zheng M, Fei F, Li C, Du J, Liu K, et al. EMT-related protein expression in polyploid giant cancer cells and their daughter cells with different passages after triptolide treatment. Medical oncology (Northwood, London, England). 2019;36(9):82.

45. Abedi M, Gheisari Y. Nodes with high centrality in protein interaction networks are responsible for driving signaling pathways in diabetic nephropathy. PeerJ. 2015;3:e1284.

46. Rabieian R, Abedi M, Gheisari Y. Central Nodes in Protein Interaction Networks Drive Critical Functions in Transforming Growth Factor Beta-1 Stimulated Kidney Cells. Cell journal. 2017;18(4):514-31.

47. Potapova T, Gorbsky GJ. The Consequences of Chromosome Segregation Errors in Mitosis and Meiosis. Biology. 2017;6(1).

48. Salmina K, Huna A, Kalejs M, Pjanova D, Scherthan H, Cragg MS, et al. The Cancer Aneuploidy Paradox: In the Light of Evolution. Genes. 2019;10(2).

49. Jordan MA, Wilson L. Microtubules as a target for anticancer drugs. Nature reviews Cancer. 2004;4(4):253-65.

50. Zdioruk M, Want A, Mietelska-Porowska A, Laskowska-Kaszub K, Wojsiat J, Klejman A, et al. A New Inhibitor of Tubulin Polymerization Kills Multiple Cancer Cell Types and Reveals p21-Mediated Mechanism Determining Cell Death after Mitotic Catastrophe. Cancers. 2020;12(8).

51. Niu N, Zhang J, Zhang N, Mercado-Uribe I, Tao F, Han Z, et al. Linking genomic reorganization to tumor initiation via the giant cell cycle. Oncogenesis. 2016;5(12):e281.

52. Wang Q, Lu F, Lan R. RNA-sequencing dissects the transcriptome of polyploid cancer cells that are resistant to combined treatments of cisplatin with paclitaxel and docetaxel. Molecular bioSystems. 2017;13(10):2125-34.

53. Yuan B, Hao J, Zhang Q, Wang Y, Zhu Y. Role of Bcl-2 on drug resistance in breast cancer polyploidyinduced spindle poisons. Oncology letters. 2020;19(3):1701-10.

54. Mujagic H, Chen SS, Geist R, Occhipinti SJ, Conger BM, Smith CA, et al. Effects of vincristine on cell survival, cell cycle progression, and mitotic accumulation in asynchronously growing Sarcoma 180 cells. Cancer research. 1983;43(8):3591-7.

\section{Figures}


A

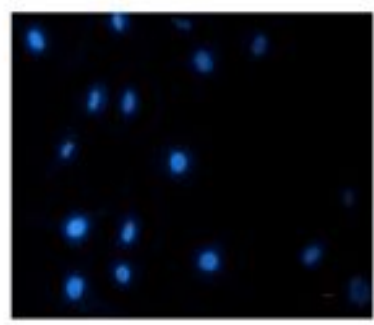

D

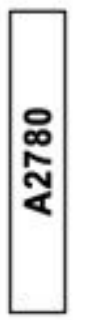

Cisplatin

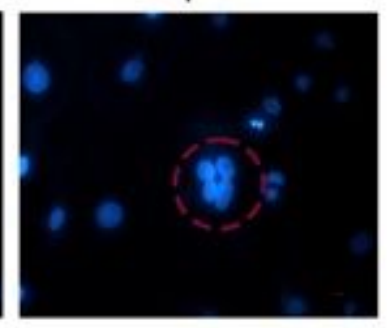

Cisplatin

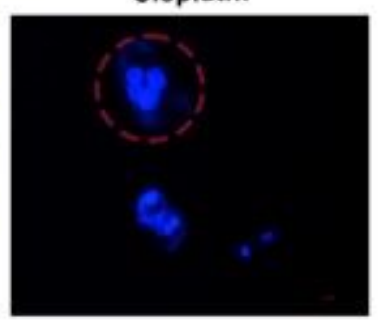

B

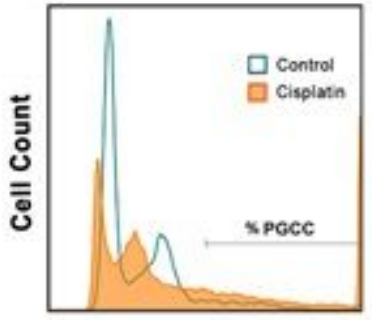

DNA content

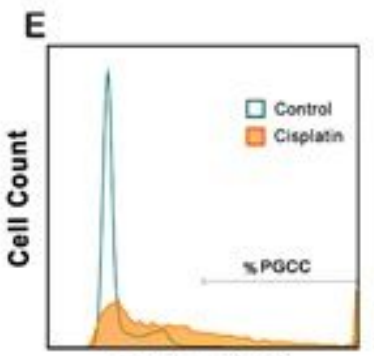

DNA content
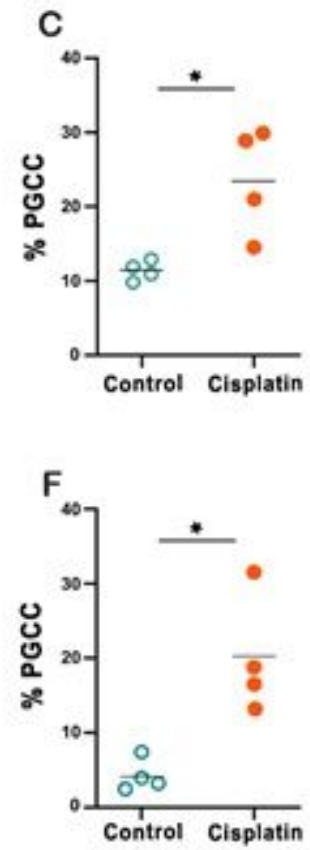

Figure 1

Augmentation of polyploid cells in SCOV-3 and A2780 cell lines post cisplatin treatment. a, $d$ Fluorescent images of the cells in test and control group (DAPI staining) after 72 hours cisplatin treatment and 7 days recovery. Scale bars: $50 \mu \mathrm{m}$. b, e DNA content analysis before and after cisplatin treatment. c, f The percentage of PGCCs in 4 replicates of each group. * $p$-value $<0.05$, black line $=$ Mean. Significance was calculated by unpaired Mann-Whitney t-test.

A

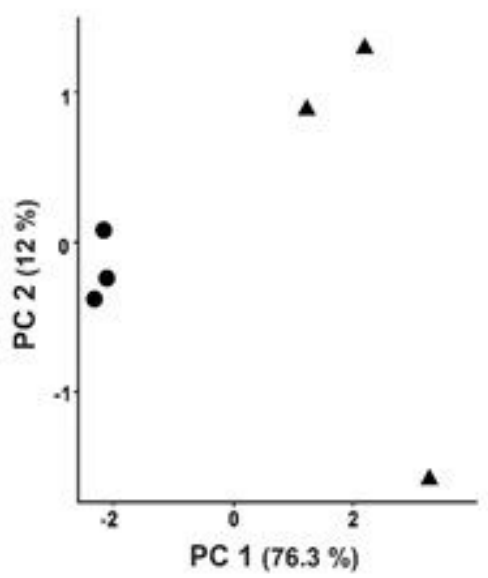

B

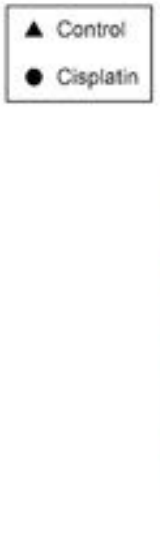

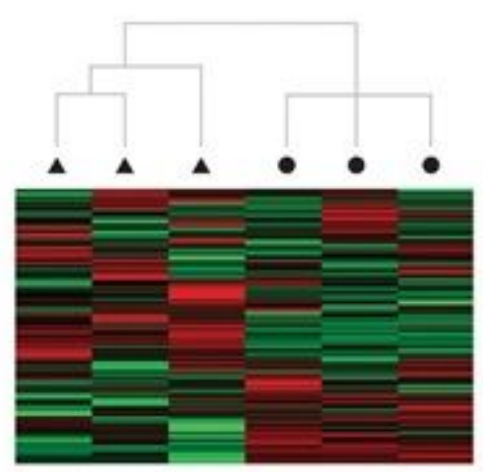

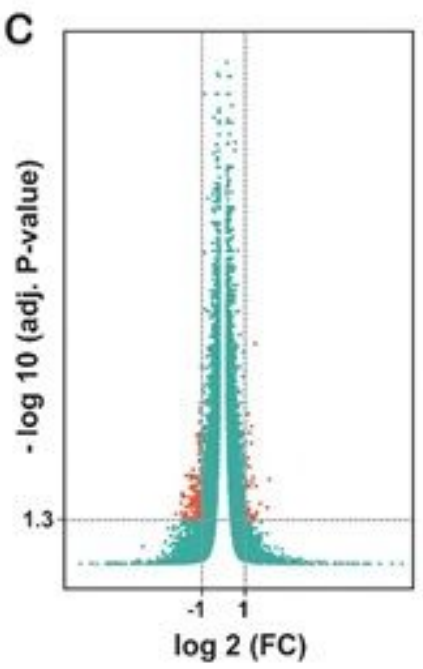

\section{Figure 2}

Quality assessment of the dataset. a, b Principal component analysis and hierarchical clustering could successfully separate control and cisplatin treatment groups. c The Volcano plot demonstrates the genes with adjusted $\mathrm{p}$-value $<0.05$ and $|\log \mathrm{FC}| \geq 1$ considered as DEGs in this study (orange dots). 
A

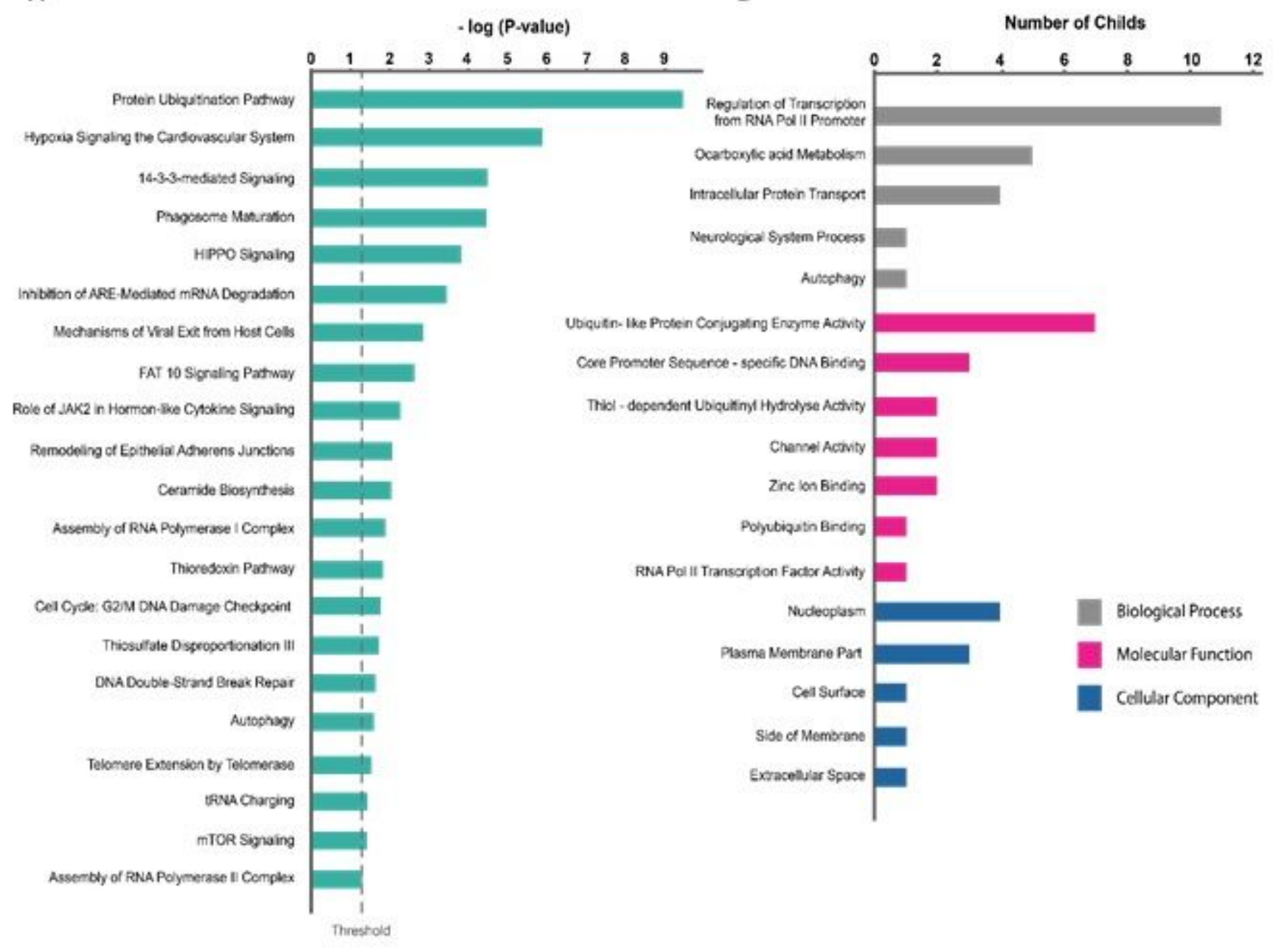

\section{Figure 3}

Pathway and gene ontology enrichment analysis determined activation of nuclear processes in the resistant cells. a Pathway enrichment analysis and, b gene ontology enrichment for biological process, molecular function and cellular component (The horizontal axis shows the number of Childs of each parental term). In both of these analysis p-value $<0.05$ was considered as the significance threshold. 


\begin{tabular}{|ll|}
\hline \multicolumn{2}{|c|}{ Top Central DE genes } \\
\hline Degree & Betweenness \\
\hline UBC & UBC \\
POLR2C & POLR2C \\
RANBP2 & PLK1 \\
PLK1 & PIK3CA \\
NUP85 & UPF3B \\
PPP2CB & SYNJ1 \\
UPF3B & PPP2CB \\
RPS7 & SP1 \\
ELOB & SUMO1 \\
UBE2D2 & RAB1B \\
\hline
\end{tabular}

B

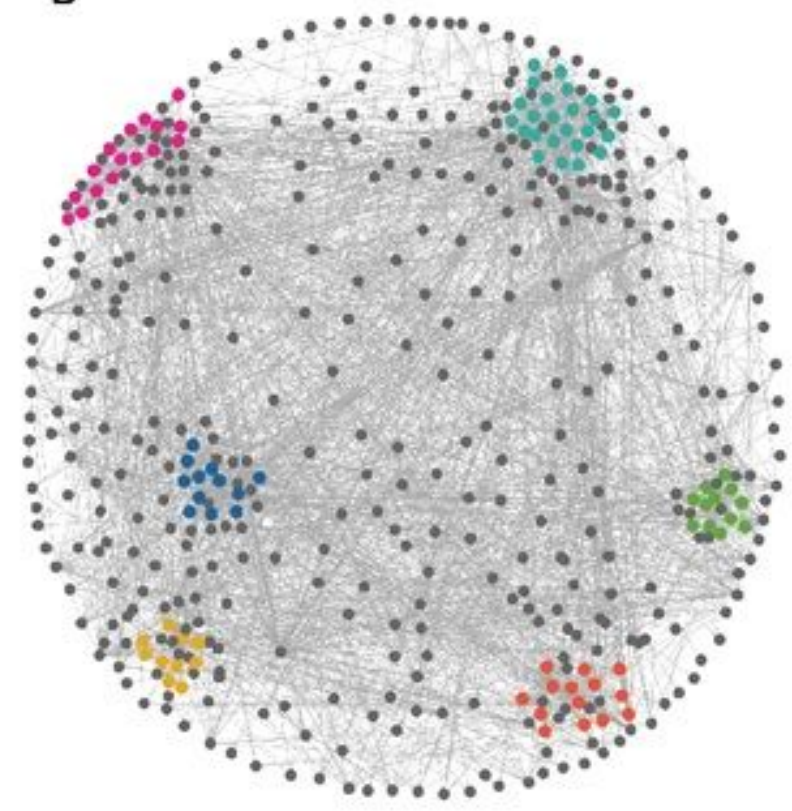

mRNA splicing

Protein export from nucleus

Protein localization to ER

snRNA metabolism

Negative regulation of chromatid segregation

Microtubole polymerization

Membrane budding

\section{Figure 4}

Top central DEGs and structural modules of the PPI network of cisplatin-resistant cells were determined. a Centrality analysis was performed and top 10 genes with the highest degree and betweenness centrality measures were detected. b The 6 high-rank modules of the network (based on clustering coefficient centrality) and their ontology (biological process) were determined. 